\title{
Soft $\zeta$-Rough Set and Its Applications in Decision Making of Coronavirus
}

\author{
M. A. El Safty ${ }^{1, *}$, Samirah Al Zahrani ${ }^{1}$, M. K. El-Bably ${ }^{2}$ and M. El Sayed ${ }^{3}$ \\ ${ }^{1}$ Department of Mathematics and Statistics, College of Science, Taif University, Taif, 21944, Saudi Arabia \\ ${ }^{2}$ Department of Mathematics, Faculty of Science, Tanta University, Tanta, Egypt \\ ${ }^{3}$ Department of Mathematics, College of Science and Arts, Najran University, Najran, 66445, Saudi Arabia \\ *Corresponding Author: M. A. El Safty. Email: m.elsafty@tu.edu.sa \\ Received: 09 April 2021; Accepted: 10 May 2021
}

\begin{abstract}
In this paper, we present a proposed method for generating a soft rough approximation as a modification and generalization of Zhaowen et al. approach. Comparisons were obtained between our approach and the previous study and also. Eventually, an application on Coronavirus (COVID-19) has been presented, illustrated using our proposed concept, and some influencing results for symptoms of Coronavirus patients have been deduced. Moreover, following these concepts, we construct an algorithm and apply it to a decision-making problem to demonstrate the applicability of our proposed approach. Finally, a proposed approach that competes with others has been obtained, as well as realistic results for patients with Coronavirus. Moreover, we used MATLAB programming to obtain the results; these results are consistent with those of the World Health Organization and an accurate proposal competing with the method of Zhaowen et al. has been studied. Therefore, it is recommended that our proposed concept be used in future decision making.
\end{abstract}

Keywords: Soft set; soft rough set; soft $\zeta$ rough set; COVID-19; intelligence discovery; decision making

\section{Introduction}

In 1999 Molodtsov [1] have introduced the soft set notion and progressing basics of this theory as a new diverse for modeling roughness and uncertainties. Diverse fields of applications of his approach were used in solving many practical problems in economics, engineering, social science, medical science... etc. Researchers have implementing various kinds of soft, rough and fuzzy sets (see [1-15]).

Often, the right decision making for many real-life issues is very difficult in our daily lives, which is highly essential for choosing the best solution to our discussions. Therefore, we have to consider various features in order to produce the highest practical solution to these problems. For this cause, we use the chosen mathematical instrument in the current article, namely soft rough set theory, in decision making. Decision making application was applied by Maji et al. [10,11]. Using soft set approach and accordingly they expand this approach to fuzzy soft set theory in [13]. Soft rough model was defined by [15].

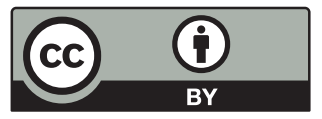

This work is licensed under a Creative Commons Attribution 4.0 International License, which permits unrestricted use, distribution, and reproduction in any medium, provided the original work is properly cited. 
Coronavirus emerged in 2019, in Wuhan, China. This virus is a new strain that has not been previously identified in humans. It was believed that Coronaviruses spread from dirty, dry surfaces, like automatic mucous membrane pollination in the nose, eyes, or mouth, reinforcing the importance of a clear understanding of the persistence of coronaviruses on inanimate surfaces [16]. Therefore, two factors which are in contact with infected surfaces and encounters with infected viruses, affect the transmission. As a result, many scientific papers have been published and many researchers have studied this virus, such as ([16-22]).

As a generalization to Pawlak's rough models [23]. Based on this structure, they defined soft rough approximations, soft rough sets and some related concepts, such as ([23-27]).

The main objective of our belief is to have a certain influence on the continuous approximation of such basic mathematical principles and to provide a modern method for computational mathematics of real-life problems. In fact, it considers latest generalized soft, rough approximations, called soft $\zeta$-rough approximations, are defined as a generalization to Zhaowen et al. [15] approximations and their properties are studied. We will prove that our approaches are more accurate and general from Zhaowen et al. approaches. The importance of the current approximations is not only that it is reducing or deleting the boundary regions, but also, it's satisfying all properties of Pawlak's rough sets without any restrictions. Comparisons between our method and the method of Zhaowen et al. are obtained.

Several examples are provided to illustrate the links between topologies and relationships of the soft set. Finally, we are added three applications. in making decisions regarding our strategy. One of them represents a beginning point for apply soft rough approach to solve the problem of Coronavirus contagion. At the end of the paper, we give two an algorithm which can be used to have a decision making for information system in terms of soft $\zeta$-rough approximations.

The main programming for this paper is as follows:

Step 1: Input the set $\hat{W}$ and the set of features represent the data as an information table, rows of which are labeled by features $A$, columns by objects and entries of the table are features values.

Step 2: Compute the rough neighborhood from the information table.

Step 3: Compute the soft $\zeta$-upper approximation, $\zeta$-lower approximation and $\zeta$-boundary for the decision set $M \subseteq \hat{W}$.

Step 4: Remove a feature $a_{1}$ from the condition's features $(A)$ and then find the rough neighborhood $A-\left\{a_{1}\right\}$.

Step 5: Comparing $\zeta$-boundary for the decision set $M \subseteq \hat{W}$ on $A-\left\{a_{i}\right\}$ with Step 3.

Step 6: Repeat Steps 4 and 5 for all attributes in $A$.

Step 7: Those attributes in $A$ for which $B N D_{A-\left\{a_{i}\right\}}^{\varepsilon}(M) \neq B N D_{A}^{\varepsilon}(M)$ forms the Core $(\hat{W})$.

Finally, we explain the importance of the proposed method in the medical sciences for application in decision-making problems. In fact, a medical application has been introduced in the decision-making process of COVID-19 Medical Diagnostic Information System with the algorithm. This application may help the world to reduce the spread of Coronavirus.

The paper is structured as follows: The basic concepts of the rough set and soft set were explored in section two and three. The implementation of COVID-19 for each subclass of 
attributes in the information systems and comparative analysis was presented in section four and five. Section six concludes and highlights future scope.

\section{Preliminaries}

In this section, we give some basic definitions and results that used in sequel are mentioned.

\subsection{Pawlak Rough Set Theory}

In 1982, Pawlak [23] introduced the theory of rough set as a new mathematical methodology or easy tools in order to deal with the vagueness in knowledge-based systems, information systems and data dissection. This theory has many applications in many fields that are used to process control, economics, such as medical diagnosis, chemistry, psychology, finance, marketing, biochemistry, environmental science, intelligent agents, image analysis, biology, conflict analysis, telecommunication, and other fields (See: [23-27], and the bibliography in these papers).

Definition 2.1 [23] Assuming that $\hat{W}$ be a set, and $\Re$ be an equivalence relation on $\hat{W}$, we use $\hat{W} \backslash \Re$ the a collections of all equivalence classes of $\Re$ and $[x]_{\Re}$. It is indicated an equivalence class in $\Re$ containing an element $x \in \hat{W}$. Then, the pair $(\hat{W}, \Re)$ it's called an approximation space and for every $M \subseteq \hat{W}$, we can define the lower and upper approximation of $M$ by $\underline{\Re}(M)=$ $\left\{x \in \hat{W}:[x]_{\Re} \subseteq M\right\}$ and $\bar{\Re}(M)=\left\{x \in \hat{W}:[x]_{\Re} \cap M \neq \phi\right\}$, respectively.

According to Pawlak's definition, $M$ it's called a rough set if $\underline{R}(M) \neq \bar{\Re}(M)$.

Proposition 2.1 [23] Let $\phi$ be the empty set and $M^{c}$ be the complement of $M \subseteq \hat{W}$. Pawlak's rough sets have the next characteristic:
L1 $\Re(M) \subseteq M$
$\mathrm{U} 1 \quad M \subseteq \bar{\Re}(M)$
L2 $\Re(\phi)=\phi$
$\mathrm{U} 2 \bar{\Re}(\phi)=\phi$
L3 $\mathfrak{R}(\hat{W})=\hat{W}$
$\mathrm{U} 3 \bar{\Re}(\hat{W})=\hat{W}$
L4 $\Re(M \cap N)=\Re(M) \cap \Re(N)$
U4 $\bar{\Re}(M \cup N)=\bar{\Re}(M) \cup \bar{\Re}(N)$
L5 If $M \subseteq N$ then $\mathfrak{R}_{-}(M) \subseteq \mathfrak{R}_{-}(N)$
U5 If $M \subseteq N$ the $\bar{\Re}(M) \subseteq \bar{\Re}(N)$
L6 $\mathfrak{R}(M) \cup \Re(N) \subseteq \Re(M \cup N)$
$\mathrm{U} 6 \bar{\Re}(M \cap N) \subseteq \bar{\Re}(M) \cap \bar{\Re}(N)$
L7 $\underset{-}{\Re}\left(M^{c}\right)=(\bar{\Re}(M))^{C}$
$\mathrm{U} 7 \bar{\Re}\left(M^{c}\right)=(\underset{-}{\Re(M)})^{C}$
$\operatorname{L8} \stackrel{\Re}{\Re}\left(\mathfrak{R}_{-}(M)\right)=\underline{-}(M)$
U8 $\bar{\Re}(\bar{\Re}(M))=\bar{\Re}(M)$

L9 If $M \in \hat{W} / \Re$ then $\mathfrak{R}(M)=M$

U9 If $M \in \hat{W} / \Re$ then $\bar{\Re}(M)=M$ 


\subsection{Soft Set Theory and Soft Rough Set}

Let us recall now the soft set notion, which is a newly-emerging mathematical approach to vagueness. Let $\hat{W}$ be an initial universe of objects and $E_{W}$ (simply denoted by $E$ ) the set of certain parameters in relation to the objects in $\hat{W}$. Parameters are often attributing, characteristics, or properties of the objects in $\hat{W}$. Let $P(\hat{W})$ denote the power set of $\hat{W}$. Following the Definition 2.1 gives the concept of soft sets as follows.

Definition 2.3 [12] Let $\hat{S}=(F, A)$ be soft set over $\hat{W}$, then we define a binary relation on $\hat{W}$ by

i. $x \mathfrak{R}^{f} y \Leftrightarrow \exists e \in E,\{x, y\} \subseteq f(a)$ for each $x, y \in \hat{W}$, then $\mathfrak{R}^{f}$ is called the binary relation induced by $(F, A)$ on $\hat{W}$.

ii. For each $x \in \hat{W}$ define successor neighborhood $\left(\Re^{f}\right)_{\hat{S}}(x)=\left\{y \in \hat{W}: x \Re^{f} y\right\}$.

Definition 2.4 [15] Let $\hat{S}=(F, A)$ be a soft set over $\hat{W}$. Then the pair $A_{\hat{S}}=(\hat{W}, \hat{S})$ is called a soft approximation space, we define the soft $A_{\hat{S}}$-lower and soft $A_{\hat{S}}$-upper approximations of any subset $M \subseteq \hat{W}$ respectively by the following two operations:

$\left.\underline{\hat{S}}(M)=\left\{\hat{W} \in \hat{W}:\left(\Re^{f}\right)_{\hat{S}}(x) \subseteq M\right\} \cdot \overline{\hat{S}}(M)=\left\{\hat{W} \in \hat{W}:\left(\Re^{f}\right)_{\hat{S}}(x) \cap M \neq \phi\right]\right\}$.

Proposition 2.2 [15] Assuming that $\hat{S}=(F, A)$ be a soft set upon $\hat{W}$ and $A_{\hat{S}}=(\hat{W}, \hat{S})$ a soft approximation space. Then the soft $A_{\hat{S}}$-lower and $A_{\hat{S}}$-upper approximations of $M \subseteq \hat{W}$ :

i. $\underline{\hat{S}}(\phi)=\overline{\hat{S}}(\phi)=\phi$. if $(F, A)$ is full soft set

ii. $\underline{\hat{S}}(\hat{W})=\overline{\hat{S}}(\hat{W})=\cup_{e \in A} f(e)$. if $(F, A)$ is full soft set

iii. $\underline{\hat{S}}(M \cap N)=\underline{\hat{S}}(M) \cap \underline{\hat{S}}(N)$.

iv. $\overline{\hat{S}}(M \cup N)=\overline{\overline{\hat{S}}}(M) \cup \overline{\overline{\hat{S}}}(N)$.

v. $\underline{\hat{S}}\left(M^{c}\right)=(\overline{\hat{S}}(M))^{C}$

vi. $\overline{\hat{S}}\left(M^{c}\right)=(\underline{\hat{S}}(M))^{C}$

vii. If $M \subseteq N$, then $\underline{\hat{S}}(M) \subseteq \underline{\hat{S}}(N)$ and $\overline{\hat{S}}(M) \subseteq \overline{\hat{S}}(N)$

viii. $\overline{\hat{S}}(\underline{\hat{S}}(M) \subseteq M \subseteq(\underline{\hat{S}}(\overline{\hat{S}}(M))$

Proposition 2.3 [15] Let $\hat{S}=(F, A)$ be a soft set over $\hat{W}$ and $A_{\hat{S}}=(\hat{W}, \hat{S})$ a soft approximation space. Then:

i. If $(F, A)$ is keeping union and full soft set then $\overline{\hat{S}}_{F}(M) \supseteq \overline{\hat{S}}(M)$.

ii. If $(F, A)$ is a partition, then $\underline{\hat{S}}_{F}(M)=\underline{\hat{S}}(M)$ and $\overline{\hat{S}}_{F}(M)=\overline{\hat{S}}(M)$. 
Proposition 2.4 [15] Let $\hat{S}=(F, A)$ be a soft set over $\hat{W}$ and $A_{\hat{S}}=(\hat{W}, \hat{S})$ a soft approximation space. Then for each $M \subseteq N$ :

i. $\underline{\hat{S}}(\underline{\hat{S}}(M))=\underline{\hat{S}}(M)$.

ii. $\overline{\hat{S}}(\overline{\hat{S}}(M))=\overline{\hat{S}}(M)$.

Definition 2.5 [2] Let $\hat{S}=(F, A)$ be a soft set over $\hat{W}$ and $A_{\hat{S}}=(\hat{W}, \hat{S})$ a soft approximation space. Then, $\hat{S}$ is said to be a full soft set if $\hat{W}=\cup_{e \in A} F(e)$.

It is clear that if $\hat{S}$ is a full soft set, then $\forall x \in \hat{W}, \exists e \in A$ such that $x \in F(e)$.

Proposition 2.5 [15] Let $\hat{S}=(F, A)$ be a full soft set over $\hat{W}$ and $A_{\hat{S}}=(\hat{W}, \hat{S})$ a soft approximation space. Then, the following conditions are true:

i. $\underline{S}(\hat{W})=\overline{\hat{S}}(\hat{W})=\hat{W}$.

ii. $M \subseteq \overline{\hat{S}}(M), \forall M \subseteq \hat{W}$.

\section{Generalized Soft Rough Approximations}

In this section, we define new generalized soft, rough approximations so-called soft $\xi$-rough approximations. The properties of the suggested approaches are superimposed. Relationship among our approaches and the previous one in Li et al. [15] are obtained. Many examples and counter examples are introduced. We will prove that our approach is a generalization to Pawlak [23] and Feng et al. [2] approaches.

Definition 3.1 Let $\hat{S}=(F, A)$ be a soft set over $\hat{W}$ and $A_{\hat{S}}=(\hat{W}, \hat{S})$ a soft approximation space. Then, the soft $\xi$-lower, $\xi$-upper approximations of any subset $M \subseteq \hat{W}$ respectively by: $\underline{\hat{S}}_{\xi}(M)=M \cap \overline{\overline{\hat{S}}}(\underline{\hat{S}}(M)), \overline{\hat{S}}_{\xi}(M)=M \cup \overline{\hat{S}}(\underline{\hat{S}}(M))$. In general, we refer to $\underline{\hat{S}}_{\xi}(M)$ and $\overline{\hat{S}}_{\xi}(M)$ as soft $\xi$-rough approximations of $M \subseteq \hat{W}$ with respect to $A_{\hat{S}}$.

Definition 3.2 Let $A_{\hat{S}}=(\hat{W}, \hat{S})$ be a soft approximation space and $M \subseteq \hat{W}$. Then, the soft $\xi$-positive, $\xi$-negative, $\xi$-boundary regions and the $\xi$-accuracy of the soft $\xi$-approximations are defined respectively by:

$$
\begin{aligned}
& \quad P O \hat{S}_{\xi}(M)=\underline{\hat{S}}_{\xi}(M), N E G_{\zeta}(M)=\hat{W}-\overline{\hat{S}}_{\xi}(M), B N D_{\xi}(M)=\overline{\hat{S}}_{\xi}(M)-\underline{\hat{S}}_{\xi}(M), \text { and } \mu_{\xi}(M)= \\
& \frac{\left|\underline{\hat{S}}_{\xi}(M)\right|}{\left|\overline{\hat{S}}_{\xi}(M)\right|} \text {, where } \overline{\hat{S}}_{\xi}(M) \neq \phi .
\end{aligned}
$$

Clearly, if $\overline{\hat{S}}_{\xi}(M)=\underline{\hat{S}}_{\xi}(M)$, i.e., $B N D_{\xi}(M)=\phi$ and $\mu_{\xi}(M)=0$. Then $M \subseteq \hat{W}$ is said to be soft $\xi$-definable or soft $\xi$-exact set; otherwise $M$ is called a soft $\xi$-rough set. 
The main goal of the following results is to introduce and studied the basic properties of soft $\xi$-rough approximations $\underline{\hat{S}}_{\xi}$ and $\overline{\hat{S}}_{\xi}$.

Example 3.1 Let $\hat{S}=(F, A)$ be a soft set over $\hat{W}$ and $A_{\hat{S}}=(\hat{W}, \hat{S})$ a soft approximation space, where, $\hat{W}=\left\{x_{1}, x_{2}, x_{3}, x_{4}, x_{5}\right\}, E=\left\{e_{1}, e_{2}, e_{3}, \ldots, e_{6}\right\}$ and $A=\left\{e_{1}, e_{2}, e_{3}, e_{4}\right\} \subseteq E$ such that $(F, A)=\left\{\left(e_{1},\left\{x_{2}\right\}\right),\left(e_{2},\left\{x_{1}, x_{4}\right\}\right),\left(e_{3},\left\{x_{3}\right\}\right),\left(e_{4},\left\{x_{1}, x_{3}\right\}\right)\right\}$ is soft set, then we have $\left(\Re^{f}\right)_{\hat{S}}\left(x_{1}\right)=$ $\left\{x_{1}, x_{3}, x_{4}\right\},\left(\Re^{f}\right)_{\hat{S}}\left(x_{2}\right)=\left\{x_{2}\right\},\left(\Re^{f}\right)_{\hat{S}}\left(x_{3}\right)=\left\{x_{1}, x_{3}\right\},\left(\Re^{f}\right)_{\hat{S}}\left(x_{4}\right)=\left\{x_{1}, x_{4}\right\}$ and $\left(\Re^{f}\right)_{\hat{S}}\left(x_{5}\right)=\phi$. Let $M=\left\{x_{1}, x_{3}, x_{5}\right\}$ then we get $\underline{\hat{S}}_{\xi}(M)=\left\{x_{1}, x_{3}, x_{5}\right\}$ and $\overline{\hat{S}}_{\xi}(M)=\left\{x_{1}, x_{3}, x_{4}, x_{5}\right\}, \mu_{\xi}(M)=$ $\frac{3}{4}, B N D_{\xi}(M)=\left\{x_{4}\right\}, P O \hat{S}_{\xi}(M)=\left\{x_{1}, x_{3}, x_{5}\right\}$ and $N E G_{\xi}(M)=\left\{x_{2}\right\}$.

Proposition 3.1 Let $\hat{S}=(F, A)$ be a soft set over $\hat{W}$ and $A_{\hat{S}}=(\hat{W}, \hat{S})$ a soft approximation space. Then, the soft $\xi$-lower and $\xi$-upper approximations of $M \subseteq \hat{W}$ satisfy the following properties:

i. If $M \subseteq N$, then $\underline{\hat{S}}_{\xi}(M) \subseteq \underline{\hat{S}}_{\xi}(N)$.

ii. If $M \subseteq N$, then $\overline{\hat{S}}_{\xi}(M) \subseteq \overline{\hat{S}}_{\xi}(N)$.

iii. $\underline{\hat{S}}_{\xi}(M \cap N)=\underline{\hat{S}}_{\xi}(M) \cap \underline{\hat{S}}_{\xi}(N)$.

iv. $\underline{\hat{S}}_{\xi}(M \cup N) \supseteq \underline{\hat{S}}_{\xi}(M) \cup \underline{\hat{S}}_{\xi}(N)$.

v. $\overline{\hat{S}}_{\xi}(M \cap N) \subseteq \overline{\hat{S}}_{\xi}(M) \cap \overline{\hat{S}}_{\xi}(N)$.

vi. $\overline{\hat{S}}_{\xi}(M \cup N)=\overline{\hat{S}}_{\xi}(M) \cup \overline{\hat{S}}_{\xi}(N)$.

Proof

i. Since $\underline{S}(M) \subseteq \underline{S}(N)$ and $\overline{\hat{S}}(M) \subseteq \overline{\hat{S}}(N)$ for each $M \subseteq N$. Then, for each $M \subseteq N, \underline{\hat{S}}_{\xi}(M)=$ $M \cap \overline{\hat{S}}(\underline{S}(M)) \subseteq N \cap \overline{\hat{S}}(\underline{S}(N))=\underline{\hat{S}}_{\xi}(N)$.

ii. Since $\overline{\hat{S}}(M) \subseteq \overline{\hat{S}}(N)$ for each $M \subseteq N$. Then, $\overline{\hat{S}}_{\xi}(M)=M \cup \overline{\hat{S}}(\underline{S}(M)) \subseteq N \cap \overline{\hat{S}}(\underline{S}(N))=$ $\overline{\hat{S}}_{\xi}(N)$.

iii. Since $(M \cap N) \subseteq N$, and $(M \cap N) \subseteq M$ then from (i) we get $\underline{\hat{S}}_{\xi}(M \cap N) \subseteq \overline{\hat{S}}_{\xi}(M) \ldots$ (ii) and $\underline{\hat{S}}_{\xi}(M \cap N) \subseteq \overline{\hat{S}}_{\xi}(N) \ldots$ (2), thus from (1), (2) we obtain $\underline{\hat{S}}_{\xi}(M \cap N) \subseteq \overline{\hat{S}}_{\xi}(M) \cap$ $\overline{\hat{S}}_{\xi}(N) \ldots$ (iii).

We shall prove that $\overline{\hat{S}}_{\xi}(M) \cap \overline{\hat{S}}_{\xi}(N) \subseteq \underline{\hat{S}}_{\xi}(M \cap N)$, let $x \notin \underline{\hat{S}}_{\xi}(M \cap N)$ this implies that $x \notin(M \cap N) \cap \overline{\hat{S}}(\underline{S}(M \cap N)$ hence $x \notin(M \cap N)$ or $x \notin \overline{\hat{S}}(\underline{S}(M \cap N)$ thus $x \notin M$ or $x \notin N$ and $x \notin \overline{\hat{S}}(\underline{S}(M)$ or $x \notin \overline{\hat{S}}(\underline{S}(N)$ thus $x \notin M$ or $x \notin \overline{\hat{S}}(\underline{S}(M)$ and $x \notin N$ or $x \notin \overline{\hat{S}}(\underline{S}(N)$. Therefore, $x \notin M \cap \overline{\hat{S}}(\underline{S}(M)$ or $x \notin N \cap \overline{\hat{S}} t(\underline{S}(N)$ thence $x \notin((M \cap \overline{\hat{S}}(\underline{S}(N)) \cap(N \cap \overline{\hat{\hat{S}}}(\underline{S}(M)))$ hence 
$x \notin \overline{\hat{S}}_{\xi}(M) \cap \overline{\hat{S}}_{\xi}(N)$ thus $\overline{\hat{S}}_{\xi}(M) \cap \overline{\hat{S}}_{\xi}(N) \subseteq \underline{\hat{S}}_{\xi}(M \cap N) \ldots$ (iv), from (iii) and (iv) we get $\underline{\hat{S}}_{\xi}(M \cup N)=\underline{\hat{S}}_{\xi}(M) \cup \underline{\hat{S}}_{\xi}(N)$.

iv. Since $M \subseteq M \cup N$ and $N \subseteq M \cup N$. Then, by (1), we have $\underline{\hat{S}}_{\xi}(M \cup N) \supseteq \underline{\hat{S}}_{\xi}(M) \cup \underline{\hat{S}}_{\xi}(N)$.

v. By similar way as (iv).

vi. By using (iv)-(v), the proof is obvious

Remark 3.1 The inclusion in the above Proposition part (iv) is not instead of to equal the following example shows this remark.

Example 3.2 Let $\hat{S}=(F, A)$ is a soft set over $\hat{W}$ and $A_{\hat{S}}=(\hat{W}, \hat{S})$ a soft $\xi$-approximation space, where $\hat{W}=\left\{x_{1}, x_{2}, x_{3}, x_{4}, x_{5}\right\}, E=\left\{e_{1}, e_{2}, e_{3}, e_{4}, \ldots, e_{6}\right\}$ and $A=\left\{e_{1}, e_{2}, e_{3}, e_{4}\right\} \subseteq$ $E$ such that $(F, A)=\left\{\left(e_{1},\left\{x_{1}\right\}\right),\left(e_{2},\left\{x_{2}, x_{4}\right\}\right),\left(e_{3},\left\{x_{3}\right\}\right),\left(e_{4},\left\{x_{5}\right\}\right)\right\}$ is partition now let then we have $\left(\Re^{f}\right)_{\hat{S}}\left(x_{1}\right)=\left\{x_{1}\right\},\left(\Re^{f}\right)_{\hat{S}}\left(x_{2}\right)=\left\{x_{2}, x_{4}\right\},\left(\Re^{f}\right)_{\hat{S}}\left(x_{3}\right)=\left\{x_{3}\right\},\left(\Re^{f}\right)_{\hat{S}}\left(x_{4}\right)=\left\{x_{2}, x_{4}\right\}$ and $\left(\Re^{f}\right)_{\hat{S}}\left(x_{5}\right)=\left\{x_{5}\right\}$, let $M=\left\{x_{1}, x_{2}, x_{3}\right\}, N=\left\{x_{3}, x_{4}, x_{5}\right\}$. Then we get $\underline{S}_{\xi}(M)=\left\{x_{3}\right\}, \overline{\hat{S}}_{\xi}(M)=$ $\left\{x_{1}, x_{2}, x_{3}\right\}, \underline{S}_{\xi}(N)=\left\{x_{3}, x_{5}\right\}$ and $\overline{\hat{S}}_{\xi}(N)=\left\{x_{3}, x_{4}, x_{5}\right\}, \underline{S}_{\xi}(M \cup N)=\hat{W}$, and $\underline{S}_{\xi}(M) \cup \underline{S}_{\xi}(N)=$ $\left\{x_{3}, x_{5}\right\} \subseteq \underline{S}_{\xi}(M \cup N)$.

Proposition 3.2 Assuming that $\hat{S}=(F, A)$ be a full soft set upon $\hat{W}$ and $A_{\hat{S}}=(\hat{W}, \hat{S})$ a soft approximation space. Then

i. $\overline{\hat{S}}_{\xi}(\phi)=\overline{\hat{S}}_{\xi}(\phi)=\phi$

ii. $\underline{\hat{S}}_{\xi}(\hat{W})=\cup_{e \in A} F(e)$ and $\overline{\hat{S}}_{\xi}(\hat{W})=\hat{W}$.

Proof

i. Since $\underline{\hat{S}}(\phi)=\overline{\hat{S}}(\phi)=\phi$. Then $\underline{\hat{S}}_{\xi}(\phi)=\phi \cap \overline{\overline{\hat{S}}}(\underline{\hat{S}}(\phi))=\phi$ and $\overline{\hat{S}}_{\xi}(\phi)=\phi \cup \overline{\hat{S}}(\underline{\hat{S}}(\phi))=\phi$.

ii. Since $\underline{S}(\hat{W})=\overline{\hat{S}}(\hat{W})=\cup_{e \in A} F(e)$, then $\underline{\hat{S}}_{\xi}(\hat{W})=\hat{W} \cap \overline{\hat{S}}(\underline{\hat{S}}(\hat{W}))=\hat{W} \cap \overline{\hat{S}}\left(\cup_{e \in A} F(e)\right)=$ $\cup_{e \in A} F(e)$ and $\overline{\hat{S}}_{\xi}(\hat{W})=\hat{W} \cup \overline{\hat{S}}(\underline{S}(\hat{W}))=\hat{W} \cup \overline{\hat{S}}\left(\cup_{e \in A} F(e)\right)=\hat{W}$.

Proposition 3.3 Assuming that $\hat{S}=(F, A)$ be a soft set upon $\hat{W}$ and $A_{\hat{S}}=(\hat{W}, \hat{S})$ a soft approximation space. Subsequently, for each $M \subseteq N$ :

i. $\underline{\hat{S}}_{\xi}\left(\overline{\hat{S}}_{\xi}(M)\right) \subseteq \overline{\hat{S}}_{\xi}(M)$.

ii. $\underline{S}_{\xi}\left(\underline{S}_{\xi}(M)\right)=\underline{\hat{S}}_{\xi}(M)$.

iii. $\underline{\hat{S}}_{\xi}(M)=\overline{\hat{S}}_{\xi}\left(\underline{\hat{S}}_{\xi}(M)\right)$.

iv. $\overline{\hat{S}}_{\xi}\left(\overline{\hat{S}}_{\xi}(M)\right)=\overline{\hat{S}}_{\xi}(M)$. 
Proof

i. Since $\underline{\hat{S}}_{\xi}\left(\overline{\hat{\hat{S}}}_{\xi}(M)\right)=\underline{\hat{S}}_{\xi}\left(M \cup \overline{\hat{S}}(\underline{\hat{S}}(M)) \subseteq\left(M \cup \overline{\hat{S}}(\underline{\hat{S}}(M))=\overline{\hat{\hat{S}}}_{\xi}(M)\right.\right.$ thus $\underline{S}_{\xi}\left(\overline{\hat{\hat{S}}}_{\xi}(M)\right) \subseteq$ $\overline{\hat{S}}_{\xi}(M)$.

ii. Obvious.

iii. Since $\overline{\hat{S}}_{\xi}(M)=M \cup \overline{\hat{S}}\left(\underline{\hat{S}}(M)\right.$, then $\overline{\hat{S}}_{\xi}\left(\underline{S}_{\xi}(M)\right)=\overline{\hat{S}}_{\xi}(M \cap \overline{\hat{S}}(\underline{\hat{S}}(M))) \supseteq(M \cap$ $\overline{\hat{S}}(\underline{\hat{S}}(M))=\underline{\hat{S}}_{\xi}(M)$ hence $\underline{S}_{\xi}(M) \subseteq \overline{\hat{S}}_{\xi}\left(\underline{S}_{\xi}(M)\right) \ldots$ (1) Conversely, we will prove that $\underline{\hat{S}}_{\xi}(M) \subseteq \overline{\hat{S}}_{\xi}\left(\underline{S}_{\xi}(M)\right)$ let $x \notin \overline{\hat{S}}_{\xi}\left(\underline{S}_{\xi}(M)\right)$ this $x \notin\left(\underline{S}_{\xi}(M) \cup \overline{\hat{S}}(\underline{\hat{S}}(M))\right)$ this $x \notin\left(\underline{S}_{\xi}(M)\right.$ and $x \notin \overline{\hat{S}}(\underline{\hat{S}}(M))$ hence $x \notin\left(\underline{S}_{\xi}(M) \cap \overline{\hat{S}}(\underline{\hat{S}}(M))\right) \subseteq x \notin(M \cap \overline{\hat{S}}(\underline{\hat{S}}(M)))$ then $x \notin$ $\underline{S}_{\xi}(M)$ and hence $\underline{\hat{S}}_{\xi}(M) \subseteq \overline{\hat{S}}_{\xi}\left(\underline{S}_{\xi}(M)\right) \ldots$ (2) thus $\underline{\hat{S}}_{\xi}(M)=\overline{\hat{S}}_{\xi}\left(\underline{\hat{S}}_{\xi}(M)\right)$.

iv. Obvious.

Remark 3.2 Note that the inclusion relations in Proposition 3.3 may be strict, as shown in Examples 3.1 and 3.2.

Example 3.3 From Example 3.1 let $M=\left\{x_{1}, x_{3}, x_{5}\right\}$. Then, we get $\underline{\hat{S}}_{\xi}(M)=\left\{x_{1}, x_{3}\right\}, \overline{\hat{S}}_{\xi}(M)=$ $\left\{x_{1}, x_{3}, x_{5}\right\}$ which implies $\overline{\hat{S}}_{\xi}(M)=\left\{x_{1}, x_{3}, x_{5}\right\}, \underline{S}_{\xi}\left(\overline{\hat{S}}_{\xi}(M)\right)=\left\{x_{1}, x_{3}\right\}$, Hence, $\overline{\hat{S}}_{\xi}(M) \neq$ $\underline{\hat{S}}_{\xi}\left(\overline{\hat{S}}_{\xi}(M)\right)$.

Proposition 3.4 Assuming that $\hat{S}=(F, A)$ be full soft set upon $\hat{W}$ and $A_{\hat{S}}=(\hat{W}, \hat{S})$ a soft approximation space. Subsequently

i. $\underline{\hat{S}}_{\xi}(\hat{W})=\hat{W}$.

ii. $\underline{\hat{S}}_{\xi}\left(\overline{\hat{S}}_{\xi}(M)\right)=\overline{\hat{S}}_{\xi}(M), \forall M \subseteq \hat{W}$.

Proposition 3.5 Assuming that $\hat{S}=(F, A)$ be soft set upon $\hat{W}$ and $A_{\hat{S}}=(\hat{W}, \hat{S})$ be a soft approximation space and $M \subseteq \hat{W}$ subsequently:

i. $\underline{\hat{S}}(M) \subseteq \underline{\hat{S}}_{\xi}(M)$

ii. $\overline{\hat{S}}(M)=\overline{\hat{S}}_{\xi}(M)$

Proof

i. Let $x \notin \underline{\hat{S}}_{\xi}(M)$ this implies that $x \notin M$ and $x \notin \underline{\hat{S}}(\overline{\hat{S}}(M))$. Thus, there exist two cases (a) $x \notin M \Rightarrow x \notin \underline{\hat{S}}(M)$

(b) $x \notin \overline{\hat{S}}(\underline{\hat{S}}(M)) \Rightarrow \exists x \in \hat{W}$ such that $\left(\Re^{f}\right)_{\hat{S}}(x) \nsubseteq \overline{\hat{S}}(M)$ hence $\left(\Re^{f}\right)_{\hat{S}}(x) \nsubseteq \underline{\hat{S}}(M)$ this implies that $x \notin \underline{\hat{S}}(M)$ and hence $\underline{\hat{S}}(M) \subseteq \underline{\hat{S}}_{\xi}(M)$. 
ii. Obvious

Corollary 3.1 Assuming that $A_{\hat{S}}=(\hat{W}, \hat{S})$ be a soft approximation space and $M \subseteq \hat{W}$ subsequently.

i. $B N D(M)_{\zeta} \subseteq B N D(M)$.

ii. $\mu(M) \leq \mu_{A_{\hat{S}}}(M)$.

Corollary 3.2 If $A_{\hat{S}}=(\hat{W}, \hat{S})$ is a soft approximation space and $M \subseteq \hat{W}$ if $M$ is a soft exact set, then it is a soft $\zeta$-exact set.

Remark 3.3 The converse of the above results is not true in general as Example 3.3 illustrated.

Example 3.4 Consider Ex..3.1 Let $M=\left\{x_{1}, x_{3}, x_{4}\right\}$ Then $\underline{S}(M)=\left\{x_{1}, x_{3}, x_{4}, x_{5}\right\}$ and $\overline{\hat{S}}(M)=$ $\left\{x_{1}, x_{3}, x_{4}\right\}$ and $B N D(M)=\left\{x_{5}\right\}$ and $\underline{\hat{S}}_{\xi}(M)=\left\{x_{1}, x_{3}, x_{4}\right\}$ and $\overline{\hat{S}}_{\xi}(M)=\left\{x_{1}, x_{3}, x_{4}\right\}, \mu_{A_{\hat{S}}}(M)=1$ and $B N D_{\zeta}(M)=\phi$ It is clear that $M$ is a soft $\zeta$-exact in our approach although it is a soft rough with respect to [15]

Example 3.5 From Example 3.1 Let $\hat{S}=(F, A)$ be a soft set over $\hat{W}$ and $A_{\hat{S}}=(\hat{W}, \hat{S})$ a soft approximation space. From this example we the following Tab. 1

Table 1: Comparison between our approach and Zhaowen approach for some soft sets

\begin{tabular}{|c|c|c|c|c|c|c|c|}
\hline$M$ & $\underline{S}(M)$ & $\overline{\hat{S}}(M)$ & $\overline{\hat{S}}(\underline{S}(M))$ & $\mu_{\hat{S}}(M)$ & $\underline{\hat{S}}_{\xi}(M)$ & $\overline{\hat{S}}_{\xi}(M)$ & $\mu_{A_{\hat{S}}}(M)$ \\
\hline,$\left.x_{4}\right\}$ & $\{x$ & $\left\{, x_{4}\right\}$ & $\left\{x_{1}\right.$, & $3 / 4$ & $\left\{x_{1}, x_{2}, x_{4}\right\}$ & $\left\{x_{1}, x_{2}, x_{4}\right\}$ & 1 \\
\hline$\left\{x_{1}, x_{3}\right\}$ & $\left\{x_{3}\right.$, & $\left\{x_{1}\right.$, & $\left\{x_{1}\right.$ & $2 / 3$ & $\left\{x_{1}\right.$ & $\left\{x_{1}, x_{3}\right\}$ & 1 \\
\hline$\left\{x_{1}, x_{4}\right\}$ & $\left\{x_{4}, x_{5}\right\}$ & $\left\{x_{1}\right.$, & $\left\{x_{1},\right\}$ & $2 / 3$ & $\left\{x_{1}\right.$ & $\left\{x_{1}, x_{4}\right\}$ & 1 \\
\hline$\left\{x_{1}, x_{2}, x_{4}, x_{5}\right\}$ & $\left\{x_{2}, x_{4}, x_{5}\right\}$ & $\left\{x_{1}, x_{2}, x_{3} x_{4}\right\}$ & $\left\{x_{1}, x_{2}, x_{4}\right\}$ & $3 / 4$ & $\left\{x_{1}, x_{2}, x_{4}\right\}$ & $\left\{x_{1}, x_{2}, x_{4}, x_{5}\right\}$ & $3 / 4$ \\
\hline$\left\{x_{1}\right.$ & $\left\{x_{2}, x_{3}, x_{5}\right\}$ & $\left\{x_{1}, x_{2}, x_{3}, x_{4}\right\}$ & $\left\{x_{1}, x_{2}, x_{3}\right\}$ & $3 / 4$ & $\left\{x_{1}, x_{2}, x_{3}\right\}$ & $\left\{x_{1}, x_{2}, x_{3}\right\}$ & 1 \\
\hline$\left\{x_{1}, x_{3}\right\}$ & $\left\{x_{3}, x_{5}\right\}$ & $\left\{x_{1}, x_{3} x_{4}\right\}$ & $\left\{x_{1}, x_{3}\right\}$ & $2 / 3$ & s & $\left\{x_{1}, x_{3} x_{5}\right\}$ & $2 / 3$ \\
\hline
\end{tabular}

From the above Tab. 1, we deduce our method is better than Zhaowen method [15]. Also, from the above Tab. 1, we get the following Tab. 2,

Table 2: Comparison between boundaries of Zhaowen method and our method

\begin{tabular}{lll}
\hline$M$ & Zhaowen “ $B N D_{A_{\hat{S}}}(M) "$ & Our method “ $B N D_{\xi}(M) "$ \\
\hline$\left.x_{1}, x_{2}, x_{4}\right\}$ & $\left\{x_{1}, x_{3}\right\}$ & $\phi$ \\
$\left\{x_{1}, x_{3}\right\}$ & $\left\{x_{1}, x_{4}\right\}$ & $\phi$ \\
$\left\{x_{1}, x_{4}\right\}$ & $\left\{x_{1}, x_{3}\right\}$ & $\phi$ \\
$\left\{x_{1}, x_{2}, x_{4}, x_{5}\right\}$ & $\left\{x_{1}, x_{3}\right\}$ & $\left\{x_{5}\right\}$ \\
$\left\{x_{1}, x_{2}, x_{3}\right\}$ & $\left\{x_{1}, x_{4}\right\}$ & $\phi$ \\
$\left\{x_{1}, x_{3}, x_{5}\right\}$ & $\left\{x_{1}, x_{3}\right\}$ & $\left\{x_{5}\right\}$ \\
\hline
\end{tabular}


Proposition 3.6 Assuming that $\hat{S}=(F, A)$ be a full soft set and $A_{\hat{S}}=(\hat{W}, \hat{S})$ be a soft approximation space. Subsequently, $\underline{S}_{\xi}\left(\overline{\hat{S}}_{\xi}(M)\right)=\overline{\hat{S}}_{\xi}(M), \forall M \subseteq \hat{W}$.

i. Firstly, by Proposition 3.3, we get $\underline{\hat{S}}_{\xi}\left(\overline{\hat{S}}_{\xi}(M)\right) \subseteq \overline{\hat{S}}_{\xi}(M), \forall M \subseteq \hat{W}$. Thus, we must prove the inverse relation $\overline{\hat{S}}_{\xi}(M) \subseteq \underline{\hat{S}}_{\xi}\left(\overline{\hat{S}}_{\xi}(M)\right)$ as follows:

let $\underline{\hat{S}}_{\xi}\left(\overline{\hat{S}}_{\xi}(M)\right)=(M \cup \overline{\hat{S}}(\underline{\hat{S}}(M))) \cap \overline{\hat{S}}(\underline{\hat{S}}(M \cup \overline{\hat{S}}(\underline{\hat{S}}(M))) \supseteq(M \cup \overline{\hat{S}}(\underline{\hat{S}}(M))) \cap(\underline{\hat{S}}(M \cup \overline{\hat{S}}(\underline{\hat{S}}(M)))$ $=(M \cup \overline{\hat{S}}(\underline{S}(M)))=\overline{\hat{S}}_{\xi}(M)$ then $\overline{\hat{S}}_{\xi}(M) \subseteq \underline{\hat{S}}_{\xi}\left(\overline{\hat{S}}_{\xi}(M)\right)$ thus $\underline{\hat{S}}_{\xi}\left(\overline{\hat{S}}_{\xi}(M)\right)=\overline{\hat{S}}_{\xi}(M)$.

Remark 3.4 Assuming that $\hat{S}=(F, A)$ be soft set over $\hat{W}$ and $A_{\hat{S}}=(\hat{W}, \hat{S})$ be a soft approximation space. If $(F, A)$ is soft set then

i. $\overline{\hat{S}}_{\xi}\left(M^{c}\right)=\left(\underline{\hat{S}}_{\xi}(M)\right)^{C}$, for each $M \subseteq \hat{W}$.

ii. $\underline{\hat{S}}_{\xi}\left(M^{c}\right)=\left(\overline{\hat{S}}_{\xi}(M)\right)^{C}$, for each $M \subseteq \hat{W}$.

Proposition 3.7 Assuming that $\hat{S}=(F, A)$ be soft set upon $\hat{W}$ and $A_{\hat{S}}=(\hat{W}, \hat{S})$ be a soft approximation space. If $(F, A)$ is soft set and keeping union and intersection, then for any $M \subseteq \hat{W}$, subsequently.

i. $\overline{\hat{S}}(M) \subseteq \overline{\hat{S}}_{\xi}(M)$.

ii. $\overline{\hat{S}}_{\xi}(M)=\hat{W}$.

Proposition 3.8 Let $\hat{S}=(F, A)$ be soft set over $\hat{W}$ and $A_{\hat{S}}=(\hat{W}, \hat{S})$ be a soft approximation space. If $(F, A)$ is partition, then for any $M \subseteq \hat{W}$.

i. $\underline{\hat{S}}(M)=\underline{\hat{S}}_{\xi}(M)$.

ii. $\overline{\hat{S}}_{\xi}(M) \subseteq \overline{\hat{S}}(M)$.

Proposition 3.9 Assuming that $\hat{S}=(F, A)$ be soft set upon $\hat{W}$ while $A_{\hat{S}}=(\hat{W}, \hat{S})$ be a soft approximation space. If $(F, A)$ is full soft, then for every $M \subseteq \hat{W}$ is soft $\xi$-definable if and only if $\overline{\hat{S}}_{\xi}(M)=M$.

Proof

Assume that $M$ is $\xi$-definable then $\overline{\hat{S}}_{\xi}(M)=\underline{\hat{S}}_{\xi}(M) \subseteq M$ but $M \subseteq \overline{\hat{S}}_{\xi}(M)$ hence $\overline{\hat{S}}_{\xi}(M)=M$, conversely, suppose that $\overline{\hat{S}}_{\xi}(M)=M$ this tends to $\overline{\hat{S}}_{\xi}(M)=M \cup \overline{\hat{S}}(\underline{\hat{S}}(M))=M$ thus $\overline{\hat{S}}_{\xi}(M) \subseteq M$, but $\underline{\hat{S}}_{\xi}(M) \subseteq M \subseteq \overline{\hat{S}}_{\xi}(M) \ldots$ (1). To prove that $\overline{\hat{S}}_{\xi}(M) \subseteq \underline{\hat{S}}_{\xi}(M)$, let $x \notin \underline{\hat{S}}_{\xi}(M)$ 
thus $x \notin M \cap \overline{\hat{S}}(\underline{S}(M))$ this imply that $x \notin M$ or $x \notin \underline{\hat{S}}(\overline{\hat{S}}(M))$ therefore $x \in M \cup \overline{\hat{S}}(\underline{\hat{S}}(M))$ and hence $x \notin \overline{\hat{S}}_{\xi}(M)$ thus $\overline{\hat{S}}_{\xi}(M) \subseteq \underline{\hat{S}}_{\xi}(M) \ldots$ (2), then we get from (1), (2) $\overline{\hat{S}}_{\xi}(M)=\underline{\hat{S}}_{\xi}(M)=M$. Thus $M$ is soft $\xi$-definable.

\section{Relationship Between Our Method and the Pawlak Approximation}

In this section, we shall compare between current method and the method of Pawlak.

Definition 4.1 [15] If $\Re$ is equivalence relation on $\hat{W}$ define a mappings $f^{\Re}: E \rightarrow P(M)$ by $f^{\Re}(e)=[e]_{\Re}$ for any $e \in E$ and $E=\hat{W}$ consequently, $\left(f^{\Re}\right)_{E}$ is called soft set induced by $\Re$ on $\hat{W}$.

Theorem 4.1 Let $\hat{S}=(F, A)$ be soft set over $\hat{W}$ and $A_{\hat{S}}=(\hat{W}, \hat{S})$ be a soft approximation space. If $(F, A)$ is partition, then for any $M \subseteq \hat{W}$. Then

i. $\underline{\Re}(M)=\underline{\hat{S}}(M)=\underline{\hat{S}}_{\xi}(M)$.

ii. $\bar{\Re}(M)=\overline{\hat{S}}(M)=\overline{\hat{S}}_{\xi}(M)$.

Remark 4.1 Propositions 3.1, 3.2 and 3.4 represent one of the deviations between our approach and in [15] approach. By this proposition, our approximations satisfied most of Pawlak's properties and then Tab. 3, summarize these properties and give first comparison among our method and [15] method. We then list codes in Tab. 3 to show whether these approximations satisfy the properties (L1) to (U9). In Tab. 3, the number 1 denotes yes and 0 denotes not.

Table 3: Properties of soft rough and soft $\xi$-rough approximations

\begin{tabular}{llllll}
\hline Lower & $\underline{\hat{S}}$ & $\underline{\hat{S}}_{\zeta}$ & Upper & $\overline{\hat{S}}$ & $\overline{\hat{S}}_{\zeta}$ \\
\hline$L_{1}$ & 0 & 1 & $U_{1}$ & 0 & 1 \\
$L_{2}$ & 0 & 1 & $U_{2}$ & 0 & 1 \\
$L_{3}$ & 1 & 0 & $U_{3}$ & 1 & 1 \\
$L_{4}$ & 1 & 1 & $U_{4}$ & 1 & 1 \\
$L_{5}$ & 1 & 1 & $U_{5}$ & 1 & 1 \\
$L_{6}$ & 0 & 1 & $U_{6}$ & 1 & 1 \\
$L_{7}$ & 0 & 0 & $U_{7}$ & 1 & 0 \\
$L_{8}$ & 0 & 1 & $U_{8}$ & 0 & 1 \\
$L_{9}$ & 0 & 0 & $U_{9}$ & 0 & 1 \\
\hline
\end{tabular}

The main goal of the following results is to illustrate the relationship between soft rough approximations (that given by Wang et al. [16]) and soft pre-rough approximations (that given by our approach in the present paper).

Definition 4.2 Assuming that $\hat{S}=(F, A)$ be a full soft set upon $\hat{W}, A_{\hat{S}}=(\hat{W}, \hat{S})$ a soft approximation space and $M \subseteq \hat{W}$. Then, we define the next four main types of soft $\xi$-rough sets:

i. $M$ is roughly soft $\xi$-definable if $\underline{\hat{S}}_{\xi}(M) \neq \phi$ while $\overline{\hat{S}}_{\xi}(M) \neq \hat{W}$. 
ii. $M$ is internally soft $\xi$-indefinable if $\underline{\hat{S}}_{\xi}(M)=\phi$ while $\overline{\hat{S}}_{\xi}(M) \neq \hat{W}$.

iii. $M$ is externally soft $\xi$-indefinable if $\underline{\hat{S}}_{\xi}(M) \neq \phi$ while $\overline{\hat{S}}_{\xi}(M)=\hat{W}$.

iv. $M$ is totally soft $\xi$-indefinable if $\underline{\hat{S}}_{\xi}(M)=\phi$ while $\overline{\hat{S}}_{\xi}(M)=\hat{W}$.

The intuitive meaning of this classification is as follows:

-If $M$ is roughly soft $\xi$-definable, this suggests that we are able to decide about some elements of $\hat{W}$ that they belong to $M$, and for some $U$ elements, while, we can decide that they belong to $M^{c}$, by using the knowledge available of the soft approximation space $A_{\hat{S}}$.

-If $M$ is internally soft $\xi$-indefinable, this suggests that we are able to decide about some elements of $\hat{W}$ that they belong to $M^{c}$, but we are incapable to decide for any element of $\hat{W}$ that it belongs to $M$, by employing $A_{\hat{S}}$.

-If $M$ is externally soft $\xi$-indefinable, this suggests that we are able to decide about some elements of $\hat{W}$ which they belong to $M$, but we are incapable to decide, for any element of $\hat{W}$ that it belongs to $M^{c}$, by employing $A_{\hat{S}}$.

-If $M$ is totally soft $\xi$-indefinable, we are incapable to decide for any element of $\hat{W}$, whether it belongs to $M$ or $M^{c}$, by employing $A_{\hat{S}}$.

Theorem 4.2 Let $A_{\hat{S}}=(\hat{W}, \hat{S})$ be a soft $\xi$-approximation space and $M \subseteq \hat{W}$. Then:

i. If $M$ is roughly soft $\xi$-definable then $M$ is roughly soft $A_{\hat{S}}$-definable.

ii. If $M$ is internally soft $\xi$-definable then $M$ is internally soft $A_{\hat{S}}$-indefinable.

iii. If $M$ is externally soft $\xi$-definable then $M$ is externally soft $A_{\hat{S}}$-indefinable.

iv. If $M$ is totally soft $\xi$-indefinable then $M$ is totally soft $A_{\hat{S}}$-indefinable.

Proof: By Proposition 3.5, the proof is obvious.

Remark 4.2 Theorem 4.2 represents a one of differences between soft rough approximations (that given by [15]) and soft $\xi$-rough approximations (that given by the present paper). Moreover, it illustrates the importance of our approaches in defining the sets, for example: if $M$ is totally soft $A_{\hat{S}}$-indefinable which implies $\underline{\hat{S}}(M)=\phi$ and $\overline{\hat{S}}(M)=\hat{W}$ that is, we are incapable to decide for any element of $\hat{W}$ whether it belongs to $M$ or $M^{c}$. But, by using soft $\xi$-rough approximations, $\underline{\hat{S}}_{\xi}(M) \neq \phi$ and $\overline{\hat{S}}_{\xi}(M) \neq \hat{W}$ and then $M$ can be roughly soft $\xi$-definable Which implies that we can decide on certain elements of $\hat{W}$ which they belong to $M$, and this meant while for some elements of $\hat{W}$, we able should decide that they are belong of $M^{c}$, Through using the information obtainable from the soft approximation space $A_{\hat{S}}$.

\section{Medical Application via in Decision Making of Covid-19}

In this section, we introduce a practical example as an application of our approaches in decision making for information system about infections of Coronavirus (COVID-19). In fact, we identify deciding factors of infections for COVID-19 in humans. In this model, we find gatherings, contact with injured people, and work in hospitals is the only deciding factors for infection transmission. We conclude that staying at home and not being in contact with humans protect and against viral infection with Coronavirus. According to [18] (Human-to-Human transmissions 
have been described with incubation times between 2-10 days, facilitating its spread via droplets, contaminated hands or surfaces).

Now, we introduce the proposed method; the application can be described as follows, where the objects as in [18]: $U=\left\{x_{1}, x_{2}^{\prime}, \ldots, x_{10}\right\}$ denotes 10 listed patients, the features as $A=$ $\left\{a_{1}, a_{2}, \ldots, a_{6}\right\}=\{$ Difficulty breathing, Chest pain, Temperature, Dry cough, Headache, Loss of taste or smell $\}$ and Decision Coronavirus $\{d\}$, as follows in information was collected by the World Health Organization as well as through medical groups specializing in Coronavirus (COVID-19). Considering the following information system.

Table 4: Information's decisions data set

\begin{tabular}{|c|c|c|c|c|c|c|c|}
\hline \multirow[t]{2}{*}{ Objects } & \multicolumn{3}{|c|}{ Serious symptoms } & \multicolumn{3}{|c|}{ Most common symptoms } & \multirow[t]{2}{*}{ Decision } \\
\hline & $\begin{array}{l}\text { Difficulty } \\
\text { breathing }\end{array}$ & $\begin{array}{l}\text { Chest } \\
\text { pain }\end{array}$ & Temperature & Dry cough & Headache & $\begin{array}{l}\text { Loss of taste } \\
\text { or smell }\end{array}$ & \\
\hline$x_{1}$ & Yes & Yes & High & Yes & Yes & No & Yes \\
\hline$x_{2}$ & Yes & Yes & High & Yes & Yes & No & Yes \\
\hline$x_{3}$ & Yes & Yes & Normal & Yes & No & Yes & No \\
\hline$x_{4}$ & Yes & Yes & Normal & No & No & No & No \\
\hline$x_{5}$ & Yes & Yes & Normal & Yes & No & No & No \\
\hline$x_{6}$ & Yes & No & High & Yes & Yes & No & Yes \\
\hline$x_{7}$ & No & No & High & Yes & Yes & No & Yes \\
\hline$x_{8}$ & No & No & Normal & Yes & Yes & No & No \\
\hline$x_{9}$ & No & No & High & No & No & Yes & Yes \\
\hline$x_{10}$ & No & No & High & Yes & Yes & No & Yes \\
\hline
\end{tabular}

Table 5: Consistent part of Tab. 4

\begin{tabular}{llllllll}
\hline Objects & \multicolumn{2}{l}{ Features } & \multicolumn{2}{l}{} & Decision \\
\cline { 2 - 6 } & $a_{1}$ & $a_{2}$ & $a_{3}$ & $a_{4}$ & $a_{5}$ & $a_{6}$ & $d$ \\
\hline$x_{1}$ & 2 & 2 & 2 & 2 & 2 & 1 & 2 \\
$x_{2}$ & 2 & 2 & 2 & 2 & 2 & 1 & 2 \\
$x_{3}$ & 2 & 2 & 1 & 2 & 1 & 2 & 1 \\
$x_{4}$ & 2 & 2 & 1 & 1 & 1 & 1 & 1 \\
$x_{5}$ & 2 & 2 & 1 & 2 & 1 & 1 & 1 \\
$x_{6}$ & 2 & 1 & 2 & 2 & 2 & 1 & 2 \\
$x_{7}$ & 1 & 1 & 2 & 2 & 2 & 1 & 2 \\
$x_{8}$ & 1 & 1 & 1 & 2 & 2 & 1 & 1 \\
$x_{9}$ & 1 & 1 & 2 & 1 & 1 & 2 & 2 \\
$x_{10}$ & 1 & 1 & 2 & 2 & 2 & 1 & 2 \\
\hline
\end{tabular}

We note that, IND $(A) \neq \operatorname{IND}\left(A-\left\{a_{1}\right\}\right), \ldots$, then $a_{1}, a_{3}, a_{4}$ and $a_{6}$ are indispensable. Also, we get $a_{2}$ removed then we obtain IND $(A)=\operatorname{IND}\left(A-\left\{a_{2}\right\}\right)$, and superfluous are $a_{2}, a_{5}$. 


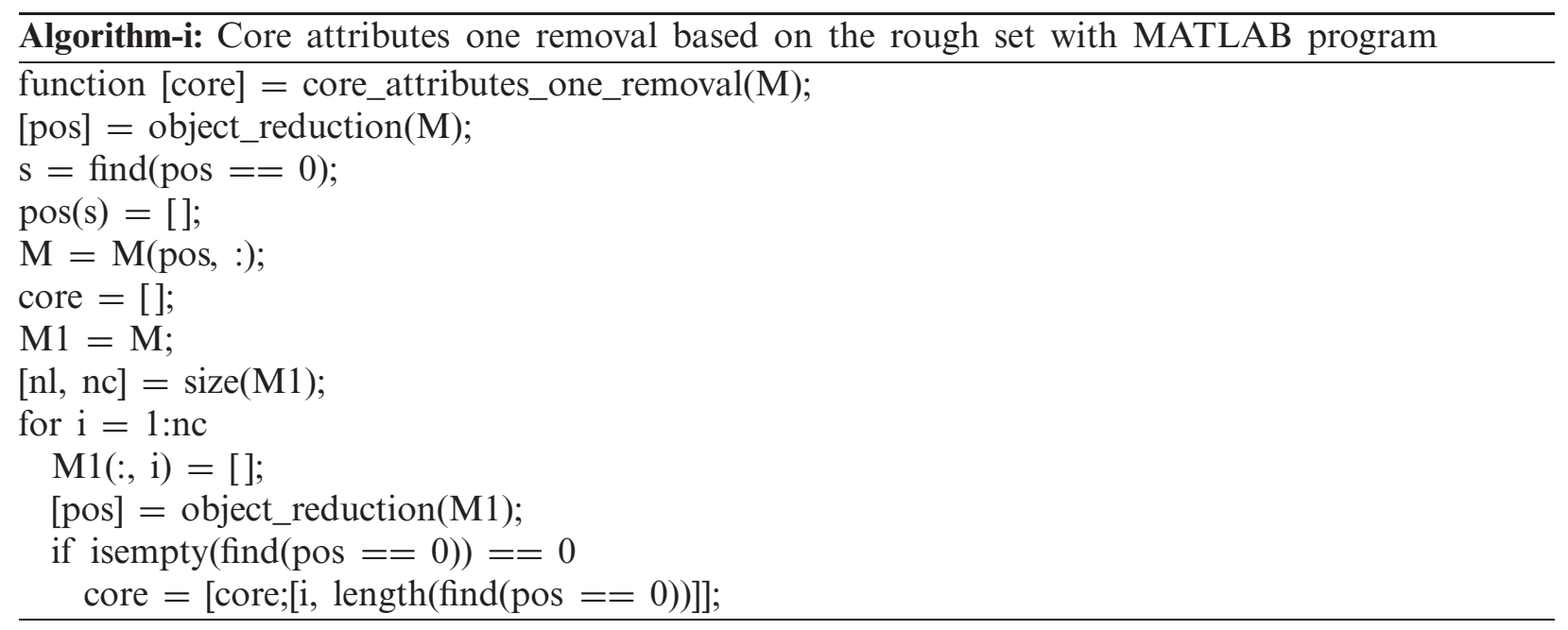

Then, we get the removal of attributes as the next Tab. 6,

Table 6: Consistent part of Tab. 5

\begin{tabular}{llllll}
\hline$U / A^{\prime}$ & \multicolumn{2}{l}{ Features $\left(A^{\prime}\right)$} & & Decision \\
\cline { 2 - 4 } & $a_{1}$ & $a_{3}$ & $a_{4}$ & $a_{6}$ & $d$ \\
\hline$x_{1}$ & 2 & 2 & 2 & 1 & 2 \\
$x_{2}$ & 2 & 2 & 2 & 1 & 2 \\
$x_{3}$ & 1 & 1 & 2 & 2 & 1 \\
$x_{4}$ & 1 & 1 & 1 & 1 & 1 \\
$x_{5}$ & 1 & 1 & 2 & 1 & 1 \\
$x_{6}$ & 2 & 2 & 2 & 1 & 2 \\
$x_{7}$ & 1 & 2 & 2 & 1 & 2 \\
$x_{8}$ & 1 & 1 & 2 & 1 & 1 \\
$x_{9}$ & 1 & 2 & 1 & 2 & 2 \\
$x_{10}$ & 1 & 2 & 2 & 1 & 2 \\
\hline
\end{tabular}

From Tab. 6, we obtain the symptoms of every patient are:

$$
\begin{aligned}
& V\left(x_{1}^{\prime}\right)=\left\{a_{1}, a_{3}, a_{4}, a_{5}, a_{6}\right\}, \quad V\left(x_{2}^{\prime}\right)=\left\{a_{1}, a_{2}, a_{3}, a_{4}, a_{5}, a_{6}\right\}, \quad V\left(x_{3}^{\prime}\right)=\left\{a_{1}, a_{2}, a_{4}, a_{6}\right\}, \\
& V\left(x_{4}^{\prime}\right)=\left\{a_{1}, a_{2}\right\}, \quad V\left(x_{5}^{\prime}\right)=\left\{a_{1}, a_{2}, a_{4}\right\}, \quad V\left(x_{6}^{\prime}\right)=\left\{a_{1}, a_{3}, a_{4}, a_{6}\right\}, \quad V\left(x_{7}^{\prime}\right)=\left\{a_{4}, a_{5}\right\}, \\
& V\left(x_{8}^{\prime}\right)=\left\{a_{4}, a_{5}\right\}, \quad V\left(x_{9}^{\prime}\right)=\phi \quad \text { and } \quad V\left(x_{10}^{\prime}\right)=\left\{a_{3}, a_{4}, a_{5}\right\} .
\end{aligned}
$$

Now, we can generate the following relation:

$$
x_{i}^{\prime} \Re x_{j}^{\prime} \Leftrightarrow V\left(x_{i}^{\prime}\right) \subseteq V\left(x_{i}^{\prime}\right) .
$$

\section{Algorithm-ii}

Step 1: Input the soft set $(F, E)$.

Step 2: Compute the right neighborhood for all elements of $\hat{W}$. 
Step 3: Investigate the soft $\zeta$-upper approximation, say, $\overline{\hat{S}}_{\zeta}(M)$ and soft $\zeta$-lower approximation, say, $\hat{S}_{\zeta}(M)$, for every $M \subseteq \hat{W}$. According to Definition 3.1.

Step 4: Determine a boundary region, say, $B N D_{\zeta}(M)$ from Step 2, for every $M \subseteq \hat{W}$. According to Definition 3.2.

Step 5: Calculate the accuracy of the approximation, say, $\mu_{\zeta}(M)$ by Step 2, for every $M \subseteq \hat{W}$. According to Definition 3.1.

Step 6: Decide, exactly, rough sets and exact sets using Definition 3.2 follows

We apply this relation for all features in the table to induce the successor neighborhoods as

$$
\begin{aligned}
\Re= & \left\{\left(x_{1}^{\prime}, x_{1}^{\prime}\right),\left(x_{1}^{\prime}, x_{2}^{\prime}\right),\left(x_{2}^{\prime}, x_{2}^{\prime}\right),\left(x_{2}^{\prime}, x_{1}^{\prime}\right),\left(x_{3}^{\prime}, x_{3}^{\prime}\right),\left(x_{4}^{\prime}, x_{4}^{\prime}\right),\left(x_{4}^{\prime}, x_{1}^{\prime}\right),\left(x_{4}^{\prime}, x_{2}^{\prime}\right),\left(x_{4}^{\prime}, x_{3}^{\prime}\right),\left(x_{4}^{\prime}, x_{5}^{\prime}\right),\right. \\
& \left(x_{5}^{\prime}, x_{5}^{\prime}\right),\left(x_{5}, x_{1}^{\prime}\right),\left(x_{5}^{\prime}, x_{2}^{\prime}\right),\left(x_{6}^{\prime}, x_{2}^{\prime}\right),\left(x_{6}^{\prime}, x_{6}^{\prime}\right),\left(x_{7}^{\prime}, x_{1}^{\prime}\right),\left(x_{7}^{\prime}, x_{2}^{\prime}\right),\left(x_{7}^{\prime}, x_{6}^{\prime}\right),\left(x_{7}^{\prime}, x_{7}^{\prime}\right),\left(x_{7}^{\prime}, x_{8}^{\prime}\right), \\
& \left(x_{7}^{\prime}, x_{9}^{\prime}\right),\left(x_{7}^{\prime}, x_{10}^{\prime}\right),\left(x_{8}^{\prime}, x_{1}^{\prime}\right),\left(x_{8}^{\prime}, x_{2}^{\prime}\right),\left(x_{8}^{\prime}, x_{6}^{\prime}\right),\left(x_{8}^{\prime}, x_{7}^{\prime}\right),\left(x_{8}^{\prime}, x_{8}^{\prime}\right),\left(x_{8}^{\prime}, x_{9}^{\prime}\right),\left(x_{8}^{\prime}, x_{10}^{\prime}\right),\left(x_{9}^{\prime}, x_{1}^{\prime}\right), \\
& \left(x_{9}^{\prime}, x_{2}^{\prime}\right),\left(x_{9}^{\prime}, x_{3}^{\prime}\right),\left(x_{9}^{\prime}, x_{4}^{\prime}\right),\left(x_{9}^{\prime}, x_{5}^{\prime}\right),\left(x_{9}^{\prime}, x_{6}^{\prime}\right),\left(x_{9}^{\prime}, x_{7}^{\prime}\right),\left(x_{9}^{\prime}, x_{8}^{\prime}\right),\left(x_{9}^{\prime}, x_{10}^{\prime}\right),\left(x_{10}^{\prime}, x_{2}^{\prime}\right), \\
& \left.\left(x_{10}^{\prime}, x_{6}^{\prime}\right),\left(x_{10}^{\prime}, x_{10}^{\prime}\right)\right\} .
\end{aligned}
$$

Thus, the successor neighborhoods of each element in $U$ of this relation are $\left(\Re^{f}\right)_{\hat{S}} x_{1}^{\prime}=$ $\left\{x_{1}, x_{2}^{\prime}\right\},\left(\Re^{f}\right)_{\hat{S}} x_{2}^{\prime}=\left\{x_{2}^{\prime}\right\},\left(\Re^{f}\right)_{\hat{S}} x_{3}^{\prime}=\left\{x_{1}, x_{2}^{\prime}, x_{3}\right\},(\Re f)_{\hat{S}} x_{4}^{\prime}=\left\{x_{1}, x_{2}^{\prime}, x_{3}, x_{4}^{\prime}, x_{5}\right\},\left(\Re^{f}\right)_{\hat{S}} x_{5}^{\prime}=$ $\left\{x_{1}, x_{2}^{\prime}, x_{3}, x_{5}\right\},\left(\Re^{f}\right)_{\hat{S}} x_{6}^{\prime}=\left\{x_{2}^{\prime}, x_{6}\right\},\left(\Re^{f}\right)_{\hat{S}} x_{7}^{\prime}=\left\{x_{1}, x_{2}^{\prime}, x_{6}, x_{7}^{\prime}, x_{8}, x_{10}\right\},\left(\Re^{f}\right)_{\hat{S}} x_{8}^{\prime}=\left\{x_{1}, x_{2}^{\prime}, x_{6}\right.$, $\left.x_{7}^{\prime}, x_{8}, x_{10}\right\},\left(\Re^{f}\right)_{\hat{S}} x_{9}^{\prime}=W$ and $\left(\Re^{f}\right)_{\hat{S}} x_{10}^{\prime}=\left\{x_{2}^{\prime}, x_{6}, x_{10}\right\}$.

Case 1: (Patients infected with COVID-19). Then the let the patients having Coronavirus is given by $M=\left\{x_{2}, x_{6}^{\prime}, x_{7}, x_{8}, x_{10}\right\}$ we have $\underset{-\varepsilon}{\hat{S}} M=M, \overline{\hat{S}}_{\varepsilon}=\hat{W}$ and the boundary of $M$ is $B N D_{A}^{\varepsilon}(M)=\left\{x_{1}, x_{3}^{\prime}, x_{4}, x_{5}, x_{9}\right\}$.

Step 1: When the features $a_{1}$-Difficulty breathing is removed: Therefore, the symptoms of every patient are: $V\left(x_{1}^{\prime}\right)=\left\{a_{2}, a_{4}, a_{5}, a_{6}\right\}, V\left(x_{2}^{\prime}\right)=\left\{a_{2}, a_{3}, a_{4}, a_{5}, a_{6}\right\}, V\left(x_{3}^{\prime}\right)=$ $\left\{a_{2}, a_{4}, a_{6}\right\}, V\left(x_{4}^{\prime}\right)=\left\{a_{2}\right\}, V\left(x_{5}^{\prime}\right)=\left\{a_{2}, a_{4}\right\}, V\left(x_{6}^{\prime}\right)=\left\{a_{3}, a_{4}, a_{5}\right\}, V\left(x_{7}^{\prime}\right)=\left\{a_{4}, a_{5}\right\}, V\left(x_{8}^{\prime}\right)=$ $\left\{a_{4}, a_{5}\right\}, V\left(x_{9}^{\prime}\right)=\phi$ and $V\left(x_{10}^{\prime}\right)=\left\{a_{3}, a_{4}, a_{5}\right\}$. Thus, the successor neighborhoods of each element in $U$ of this relation are

$\left(\Re^{f}\right)_{\hat{S}} x_{1}^{\prime}=\left\{x_{1}, x_{2}^{\prime}\right\},\left(\Re^{f}\right)_{\hat{S}} x_{2}^{\prime}=\left\{x_{2}^{\prime}\right\},\left(\Re^{f}\right)_{\hat{S}} x_{3}^{\prime}=\left\{x_{1}, x_{2}^{\prime}, x_{3}\right\},\left(\Re^{f}\right)_{\hat{S}} x_{4}^{\prime}=\left\{x_{1}, x_{2}^{\prime}, x_{3}, x_{4}^{\prime}, x_{5}\right\}$, $\left(\Re^{f}\right)_{\hat{S}} x_{5}^{\prime}=\left\{x_{1}, x_{2}^{\prime}, x_{3}, x_{5}\right\},\left(\Re^{f}\right)_{\hat{S}} x_{6}^{\prime}=\left\{x_{2}^{\prime}, x_{6}\right\},\left(\Re^{f}\right)_{\hat{S}} x_{7}^{\prime}=\left\{x_{1}, x_{2}^{\prime}, x_{6}, x_{7}^{\prime}, x_{8}, x_{10}\right\},\left(\Re^{f}\right)_{\hat{S}} x_{8}^{\prime}=$ $\left\{x_{1}, x_{2}^{\prime}, x_{6}, x_{7}^{\prime}, x_{8}, x_{10}\right\},\left(\Re^{f}\right)_{\hat{S}} x_{9}^{\prime}=W$ and $\left(\Re^{f}\right)_{\hat{S}} x_{10}^{\prime}=\left\{x_{2}^{\prime}, x_{6}, x_{10}\right\}$, then the let the patients having corona virus is given by $M=\left\{x_{2}, x_{6}^{\prime}, x_{7}, x_{8}, x_{10}\right\}$, we have $\underset{-\varepsilon}{\hat{S}} M=M, \overline{\hat{S}}_{\varepsilon}=\hat{W}$ and the boundary of $M$ is $B N D_{A-\left\{a_{1}\right\}}^{\varepsilon}(M)=\left\{x_{1}, x_{3}^{\prime}, x_{4}, x_{5}, x_{9}\right\}=B N D_{A}^{\varepsilon}(M)$.

Step 2: When the features $a_{2}$-Chest pain is removed: Thus, the successor neighborhoods of each element in $U$ of this relation are: 
$\left(\Re^{f}\right)_{\hat{S}} x_{1}^{\prime}=\left\{x_{1}, x_{2}^{\prime}\right\},\left(\Re^{f}\right)_{\hat{S}} x_{2}^{\prime}=\left\{x_{2}^{\prime}\right\},\left(\Re^{f}\right)_{\hat{S}} x_{3}^{\prime}=\left\{x_{1}, x_{2}^{\prime}, x_{3}\right\},\left(\Re^{f}\right)_{\hat{S}} x_{4}^{\prime}=\left\{x_{1}, x_{2}^{\prime}, x_{3}, x_{4}^{\prime}, x_{5}\right.$, $\left.x_{6}\right\},\left(\Re^{f}\right)_{\hat{S}} x_{5}^{\prime}=\left\{x_{1}, x_{2}^{\prime}, x_{3}, x_{5}, x_{6}\right\},\left(\Re^{f}\right)_{\hat{S}} x_{6}^{\prime}=\left\{x_{2}^{\prime}, x_{6}\right\},\left(\Re^{f}\right)_{\hat{S}} x_{7}^{\prime}=\left\{x_{1}, x_{2}^{\prime}, x_{6}, x_{7}^{\prime}, x_{8}, x_{10}\right\}$, $\left(\Re^{f}\right)_{\hat{S}} x_{8}^{\prime}=\left\{x_{1}, x_{2}^{\prime}, x_{6}, x_{7}^{\prime}, x_{8}, x_{10}\right\},\left(\Re^{f}\right)_{\hat{S}} x_{9}^{\prime}=W$ and $\left(\Re^{f}\right)_{\hat{S}} x_{10}^{\prime}=\left\{x_{2}^{\prime}, x_{6}, x_{10}\right\}$, then, the patients having Coronavirus is given by $M=\left\{x_{2}, x_{6}^{\prime}, x_{7}, x_{8}, x_{10}\right\}$, we have ${\underset{-}{\zeta}}_{\zeta}(M)=M, \overline{\hat{S}}_{\zeta}(M)=\hat{W}$ and the boundary of $M$ is $B N D_{A-\left\{a_{2}\right\}}^{\varepsilon}(M)=\left\{x_{1}, x_{3}^{\prime}, x_{4}, x_{5}, x_{9}\right\}=B N D_{A}^{\varepsilon}(M)$.

Step 3: When the features $a_{3}$-high temperature is removed: The symptoms of every patient are

$V\left(x_{1}^{\prime}\right)=\left\{a_{1}, a_{2}, a_{4}, a_{5}, a_{6}\right\}, V\left(x_{2}^{\prime}\right)=\left\{a_{1}, a_{2}, a_{4}, a_{5}, a_{6}\right\}, V\left(x_{3}^{\prime}\right)=\left\{a_{1}, a_{2}, a_{4}, a_{6}\right\}, V\left(x_{4}^{\prime}\right)=$ $\left\{a_{1}, a_{2}\right\}, V\left(x_{5}^{\prime}\right)=\left\{a_{1}, a_{2}, a_{4}\right\}, V\left(x_{6}^{\prime}\right)=\left\{a_{1}, a_{4}, a_{5}\right\}, V\left(x_{7}^{\prime}\right)=\left\{a_{4}, a_{5}\right\}, V\left(x_{8}^{\prime}\right)=\left\{a_{4}, a_{5}\right\}, V\left(x_{9}^{\prime}\right)=\phi$ and $V\left(x_{10}^{\prime}\right)=\left\{a_{4}, a_{5}\right\}$. Thus, the successor neighborhoods of each element in $U$ of this relation are

$\left(\Re^{f}\right)_{\hat{S}} x_{1}^{\prime}=\left\{x_{1}, x_{2}^{\prime}\right\},\left(\Re^{f}\right)_{\hat{S}} x_{2}^{\prime}=\left\{x_{1}, x_{2}^{\prime}\right\},\left(\Re^{f}\right)_{\hat{S}} x_{3}^{\prime}=\left\{x_{1}, x_{2}^{\prime}, x_{3}\right\}, \Re^{f}{ }_{r} x_{4}^{\prime}=\left\{x_{1}, x_{2}^{\prime}, x_{3}, x_{4}^{\prime}, x_{5}\right\}$, $\left(\Re^{f}\right)_{\hat{S}} x_{5}^{\prime}=\left\{x_{1}, x_{2}^{\prime}, x_{3}, x_{5}\right\},\left(\Re^{f}\right)_{\hat{S}} x_{6}^{\prime}=\left\{x_{1}, x_{2}^{\prime}, x_{6}\right\},\left(\Re^{f}\right)_{\hat{S}} x_{7}^{\prime}=\left\{x_{1}, x_{2}^{\prime}, x_{6}, x_{7}^{\prime}, x_{8}, x_{10}\right\},\left(\Re^{f}\right)_{\hat{S}} x_{8}^{\prime}=$ $\left\{x_{1}, x_{2}^{\prime}, x_{6}, x_{7}^{\prime}, x_{8}, x_{10}\right\},\left(\Re^{f}\right)_{\hat{S}} x_{9}^{\prime}=W$ and $\left(\Re^{f}\right)_{\hat{S}} x_{10}^{\prime}=\left\{x_{2}^{\prime}, x_{6}, x_{7}, x_{8}, x_{10}\right\}$, then, the patients having Coronavirus is given by $M=\left\{x_{2}, x_{6}^{\prime}, x_{7}, x_{8}, x_{10}\right\}$, we have $\underset{-\varepsilon}{\hat{S}} M=\phi, \overline{\hat{S}}_{\varepsilon}=M$ and the boundary of $M$ is $B N D_{A-\left\{a_{3}\right\}}^{\varepsilon}(M)=M \neq B N D_{A}^{\varepsilon}(M)$.

Step 4. When the attribute $a_{4}$-Dry cough is removed: Thus, the successor neighborhoods of each element in $U$ of this relation are:

$\left(\Re^{f}\right)_{\hat{S}} x_{1}^{\prime}=\left\{x_{1}, x_{2}^{\prime}\right\},\left(\Re^{f}\right)_{\hat{S}} x_{2}^{\prime}=\left\{x_{2}^{\prime}\right\}, \Re^{f}{ }_{r} x_{3}^{\prime}=\left\{x_{1}, x_{2}^{\prime}, x_{3}\right\},\left(\Re^{f}\right)_{\hat{S}} x_{4}^{\prime}=\left\{x_{1}, x_{2}^{\prime}, x_{3}, x_{4}^{\prime}, x_{5}\right\}$, $\left(\Re^{f}\right)_{\hat{S}} x_{5}^{\prime}=\left\{x_{1}, x_{2}^{\prime}, x_{3}, x_{5}\right\},\left(\Re^{f}\right)_{\hat{S}} x_{6}^{\prime}=\left\{x_{2}^{\prime}, x_{6}\right\},\left(\Re^{f}\right)_{\hat{S}} x_{7}^{\prime}=\left\{x_{1}, x_{2}^{\prime}, x_{6}, x_{7}^{\prime}, x_{8}, x_{10}\right\},\left(\Re^{f}\right)_{\hat{S}} x_{8}^{\prime}=$ $\left\{x_{1}, x_{2}^{\prime}, x_{6}, x_{7}^{\prime}, x_{8}, x_{10}\right\},\left(\Re^{f}\right)_{\hat{S}} x_{9}^{\prime}=W$ and $\left(\Re^{f}\right)_{\hat{S}} x_{10}^{\prime}=\left\{x_{2}^{\prime}, x_{6}, x_{10}\right\}$, then, the patients having

Coronavirus is given by $M=\left\{x_{2}, x_{6}^{\prime}, x_{7}, x_{8}, x_{10}\right\}$, we have $\underset{-\varepsilon}{\hat{S}} M=M, \overline{\hat{S}}_{\varepsilon}=W$ and the boundary of $M$ is $B N D_{A-\left\{a_{4}\right\}}^{\varepsilon}(M)=N D_{A}^{\varepsilon}(M)$.

Step 5: When the features $a_{5}$-Headache is removed: Therefore, the symptoms of every patient are:

$V\left(x_{1}^{\prime}\right)=\left\{a_{1}, a_{2}, a_{4}, a_{6}\right\}, V\left(x_{2}^{\prime}\right)=\left\{a_{1}, a_{2}, a_{3}, a_{4}, a_{6}\right\}, V\left(x_{3}^{\prime}\right)=\left\{a_{1}, a_{2}, a_{4}, a_{6}\right\}, V\left(x_{4}^{\prime}\right)=\left\{a_{1}, a_{2}\right\}$, $V\left(x_{5}^{\prime}\right)=\left\{a_{1}, a_{2}, a_{4}\right\}, V\left(x_{6}^{\prime}\right)=\left\{a_{1}, a_{3}, a_{4}\right\}, V\left(x_{7}^{\prime}\right)=\left\{a_{4}\right\}, V\left(x_{8}^{\prime}\right)=\left\{a_{4}\right\}, V\left(x_{9}^{\prime}\right)=\phi$ and $V\left(x_{10}^{\prime}\right)=$ $\left\{a_{3}, a_{4}\right\}$. Thus, the successor neighborhoods of each element in $U$ of this relation are

$$
\begin{aligned}
& \left(\Re^{f}\right)_{\hat{S}} x_{1}^{\prime}=\left\{x_{1}, x_{2}^{\prime}, x_{3}\right\},\left(\Re^{f}\right)_{\hat{S}} x_{2}^{\prime}=\left\{x_{2}^{\prime}\right\}, \Re^{f}{ }_{r} x_{3}^{\prime}=\left\{x_{1}, x_{2}^{\prime}, x_{3}\right\},\left(\Re^{f}\right)_{\hat{S}} x_{4}^{\prime}=\left\{x_{1}, x_{2}^{\prime}, x_{3}, x_{4}^{\prime}, x_{5}\right\}, \\
& \left(\Re^{f}\right)_{\hat{S}} x_{5}^{\prime}=\left\{x_{1}, x_{2}^{\prime}, x_{3}, x_{5}\right\}, \\
& \left(\Re^{f}\right)_{\hat{S}} x_{6}^{\prime}=\left\{x_{2}^{\prime}, x_{6}\right\},\left(\Re^{f}\right)_{\hat{S}} x_{7}^{\prime}=\left\{x_{1}, x_{2}^{\prime}, x_{3}, x_{6}, x_{7}^{\prime}, x_{8}, x_{10}\right\},\left(\Re^{f}\right)_{\hat{S}} x_{8}^{\prime}=\left\{x_{1}, x_{2}^{\prime}, x_{3}, x_{6}, x_{7}^{\prime}, x_{8}, x_{10}\right\}, \\
& \left(\Re^{f}\right)_{\hat{S}_{9}} x_{9}^{\prime}=W \text { and }\left(\Re^{f}\right)_{\hat{S}} x_{10}^{\prime}=\left\{x_{2}^{\prime}, x_{6}, x_{10}\right\}, \text { then, the patients having Coronavirus is given by }
\end{aligned}
$$


$M=\left\{x_{2}, x_{6}^{\prime}, x_{7}, x_{8}, x_{10}\right\}$, we have $\hat{S}_{-\varepsilon} M=M, \overline{\hat{S}}_{\varepsilon}=W$ and the boundary of $M$ is $B N D_{A-\left\{a_{5}\right\}}^{\varepsilon}(M)=$ $B N D_{A}^{\varepsilon}(M)$.

Step 6: When the features $a_{6}$-Loss of taste or smell is removed: The symptoms of every patient are:

$$
V\left(x_{1}^{\prime}\right)=\left\{a_{1}, a_{2}, a_{4}, a_{5}\right\}, V\left(x_{2}^{\prime}\right)=\left\{a_{1}, a_{2}, a_{3}, a_{4}, a_{5}\right\}, V\left(x_{3}^{\prime}\right)=\left\{a_{1}, a_{2}, a_{4}\right\}, V\left(x_{4}^{\prime}\right)=\left\{a_{1}, a_{2}\right\},
$$
$V\left(x_{5}^{\prime}\right)=\left\{a_{1}, a_{2}, a_{4}\right\}, V\left(x_{6}^{\prime}\right)=\left\{a_{1}, a_{3}, a_{4}, a_{5}\right\}, V\left(x_{7}^{\prime}\right)=\left\{a_{4}, a_{5}\right\}, V\left(x_{8}^{\prime}\right)=\left\{a_{4}, a_{5}\right\}, V\left(x_{9}^{\prime}\right)=\phi$ and $V\left(x_{10}^{\prime}\right)=\left\{a_{3}, a_{4}, a_{5}\right\}$. Thus, the successor neighborhoods of each element in $U$ of this relation are

$\left(\Re^{f}\right)_{\hat{S}} x_{1}^{\prime}=\left\{x_{1}, x_{2}^{\prime}\right\},\left(\Re^{f}\right)_{\hat{S}} x_{2}^{\prime}=\left\{x_{2}^{\prime}\right\},\left(\Re^{f}\right)_{\hat{S}} x_{3}^{\prime}=\left\{x_{1}, x_{2}^{\prime}, x_{3}, x_{5}\right\},\left(\Re^{f}\right)_{\hat{S}} x_{4}^{\prime}=\left\{x_{1}, x_{2}^{\prime}, x_{3}, x_{4}^{\prime}, x_{5}\right\}$, $\left(\Re^{f}\right)_{\hat{S}} x_{5}^{\prime}=\left\{x_{1}, x_{2}^{\prime}, x_{3}, x_{5}\right\}$,

$\left(\Re^{f}\right)_{\hat{S}} x_{6}^{\prime}=\left\{x_{2}^{\prime}, x_{6}\right\},\left(\Re^{f}\right)_{\hat{S}} x_{7}^{\prime}=\left\{x_{1}, x_{2}^{\prime}, x_{6}, x_{7}^{\prime}, x_{8}, x_{10}\right\},\left(\Re^{f}\right)_{\hat{S}} x_{8}^{\prime}=\left\{x_{1}, x_{2}^{\prime}, x_{6}, x_{7}^{\prime}, x_{8}, x_{10}\right\}$, $\left(\Re^{f}\right)_{\hat{S}} x_{9}^{\prime}=W$ and $\left(\Re^{f}\right)_{\hat{S}} x_{10}^{\prime}=\left\{x_{2}^{\prime}, x_{6}, x_{10}\right\}$, then, the patients having Coronavirus is given by

$M=\left\{x_{2}, x_{6}^{\prime}, x_{7}, x_{8}, x_{10}\right\}$, we have $\underset{-\varepsilon}{\hat{S}} M=M, \overline{\hat{S}}_{\varepsilon}=W$ and the boundary of $M$ is $B N D_{A-\left\{a_{6}\right\}}^{\varepsilon}(M)=$ $B N D_{A}^{\varepsilon}(M)$.

Hence, the CORE is: $\left\{a_{3}\right.$-Temperature $\}$, that is the impact factor for COVID-19 infection.

Case 2: (Patients are not infected with COVID-19)

The set of infected patients with $N=\left\{x_{3}, x_{4}, x_{5}, x_{9}\right\}$. By made the same steps like as Case (1), we obtain the same results. From the CORE, we observed that Temperature is the key factor for COVID-19 infection.

\section{Conclusion}

In this paper, we introduced a modification as a generalization to soft rough set models that given by Zhaowen et al., namely, Soft $\zeta$-rough approximation and study of its properties. A comparison is made between our approach and other works such as Zhaowen et al. and Pawlak. Moreover, according to our results Theorem 3.1 and its corollaries, our approach is more accurate than Zhaowen et al. approach. In addition, we used our approach in applications to Coronavirus symptoms to illustrate the relevance of our proposal in this paper, in decision making to illustrate and also to compare our method with that Zhaowen et al. approach. Also, an algorithm was obtained for our method. According to the results in Section 3 (Theorem 3.1 and Corollary 3.1, 3.2), we can say that our method is more accurate than Zhaowen et al. approach to decision making and hence this method is very useful in real-life applications. The importance of the current paper is not only that it introduces a new type of generalized soft rough set approximations, which increases the accuracy measure and reduces the boundary region of the sets which is the main aim of soft rough set, but also our approaches achieved the approximate Pawlak' rough set properties that never hold in Zhaowen et al. Finally, we provided an applied example in real-life problems to an illustrate the importance of our approaches to decision making. In fact, our proposal is helpful in deciding any future real-life problem. 
Funding Statement: This research received funding from Taif University, Researchers Supporting and Project Number (TURSP-2020/207), Taif University, Taif, Saudi Arabia.

Conflicts of Interest: The authors declare that they have no conflicts of interest to report regarding the present study.

\section{References}

[1] D. A. Molodtsov, "Soft set theory-first results," Computers \& Mathematics with Applications, vol. 37, no. 4-5, pp. 19-31, 1999.

[2] F. Feng, X. Liu, V. L. Fotea and Y. B. Jun, "Soft sets and soft rough sets," Information Sciences, vol. 181, no. 6, pp. 1125-1137, 2011.

[3] M. I. Ali and N. Rehman, "Soft dominance based rough sets with applications in information systems," International Journal of Approximate Reasoning, vol. 113, no. 1, pp. 171-195, 2019.

[4] M. I. Ali, W. K. Mi and M. Shabir, "On some new operations in soft set theory," Computers \& Mathematics with Applications, vol. 57, no. 9, pp. 1547-1553, 2009.

[5] M. I. Ali, "A note on soft sets, rough soft sets and fuzzy soft sets," Applied Soft Computing, vol. 11, no. 4, pp. 3329-3332, 2011.

[6] K. V. Babitha and J. J. Sunil, "Soft topologies generated by soft set relations," in Handbook of Research on Generalized and Hybrid Set Structures and Applications for Soft Computing. National Institute of Technology, Calicut, India, pp. 118-126, 2016. https://doi.org/10.4018/978-1-4666-9798-0.ch007.

[7] M. El Sayed, A. Al Qubati and M. K. El-Bably, "Soft pre-rough sets and its applications in decision making," Mathematical Biosciences and Engineering MBE, vol. 17, no. 5, pp. 6045-6063, 2020.

[8] M. A. El Safty, A. A. Mousa, S. A. Alblowi and M. El Sayed, "Topological approach on Fuzzy soft B-closure and B-interior and its Application in Decision Making," Nanoscience and Nanotechnology Letters, vol. 12, no. 11, pp. 1323-1328, 2020.

[9] M. A. El Safty, "Modeling uncertainty knowledge of the topological methods," Poincare Journal of Analysis \& Applications, vol. 8, no. 1, pp. 1-14, 2021.

[10] P. K. Maji, R. Biswas and A. R. Roy, "Soft set theory," Computers and Mathematics with Applications, vol. 45, no. 4-5, pp. 555-562, 2003.

[11] P. K. Maji, A. R. Roy and R. Biswas, "An application of soft sets in a decision making problem," Computers Math. Appl., vol. 44, no. 8-9, pp. 1077-1083, 2002.

[12] Q. M. Sun, Z. L. Zhang and J. Liu, "Soft sets and soft modules," Rough Sets and Knowledge Technology, vol. 5009, pp. 403-409, 2008.

[13] B. Tanay and M. B. Kandemir, "Topological structure of fuzzy soft sets," Computers and Mathematics with Application, vol. 61, no. 10, pp. 2952-2957, 2011.

[14] X. Yang, D. Yu, J. Yang and C. Wu, "Generalization of soft set theory: From crisp to fuzzy case," Fuzzy Information and Engineering, ICFIE, vol. 40, pp. 345-354, 2007.

[15] Z. Li, B. Qin and Z. Cai, "Soft rough approximation operators and related results," Journal of Applied Mathematics, vol. 2013, pp. 1-15, 2013.

[16] L. Wang, J. Wang, H. Zhao, Y. Shi, K. Wang et al., "Modelling and assessing the effects of medical resources on transmission of novel Coronavirus (COVID-19) in Wuhan," China Mathematical Biosciences and Engineering, vol. 17, no. 4, pp. 2936-2949, 2020.

[17] S. A. Alblowi, M. El Sayed and M. A. El Safty, "Decision making based on fuzzy soft sets and its application in COVID-19," Intelligent Automation \& Soft Computing, 2021.

[18] M. A. El Safty and Samirah Al Zahrani, "Topological modeling for symptom reduction of Coronavirus," Punjab Uni. J. Math., vol. 53, no. 3, pp. 47-59, 2021.

[19] G. Kampf, D. Todt, S. Pfaender and E. Steinmann, "Persistence of Coronaviruses on inanimate surfaces and their inactivation with biocidal agents," Journal of Hospital Infection, vol. 104, no. 3, pp. 246-251, 2020. 
[20] M. Naveed, M. Rafiq, A. Raza, N. Ahmed and I. Khan, "Mathematical analysis of novel Coronavirus (2019-ncov) delay pandemic model," Computers, Materials \& Continua, vol. 64, no. 3, pp. 1401-1414, 2020.

[21] A. A. Ojugo and R. E. Yoro, "Migration pattern as threshold parameter in the propagation of the COVID-19 Epidemic using an actor-based model for SI-social graph," Journal of Information and Visualization, vol. 2, no. 2, pp. 93-105, 2021.

[22] R. Patil, U. Patel and T. Sarkar, "COVID-19 cases prediction using regression and novel SSM model for non-converged countries," Journal of Applied Science, Engineering, Technology, and Education, vol. 3, no. 1, pp. 74-81, 2021.

[23] Z. Pawlak, "Rough sets," Int J. of Information and Computer Sciences, vol. 11, no. 5, pp. 341-356, 1982.

[24] M. A. El Safty and A. M. Alkhathami, "A Topological method for reduction of digital information uncertainty," Soft Computing, vol. 24, no. 1, pp. 385-396, 2020.

[25] M. A. El Safty, "Topological method for a study of discriminating three categories of banks and its use in attributes reduction," International Journal of Scientific Research in Science, Engineering and Technology, vol. 7, no. 5, pp. 221-233, 2020.

[26] M. El Sayed, Kh. Abd-Rabou and M. A. El Safty, "New classes of soft open sets in soft generalized topological spaces," The Journal of Fuzzy Mathematics, vol. 28, pp. 721-732, 2020.

[27] M. K. El-Bably, "Comparisons between near open sets and rough approximations," International Journal of Granular Computing, Rough Sets and Intelligent Systems, vol. 4, no. 1, pp. 64-83, 2015. 